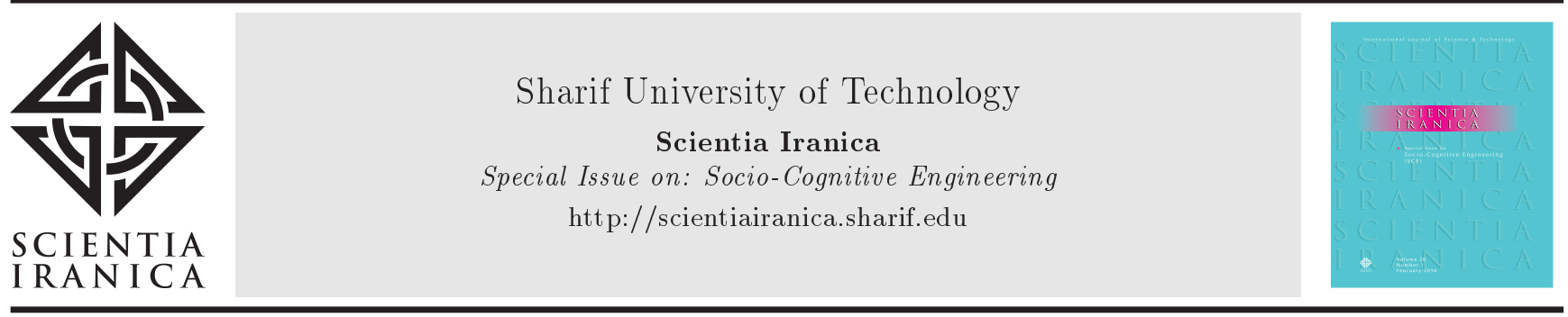

\title{
Teaching music to children with autism: A social robotics challenge
}

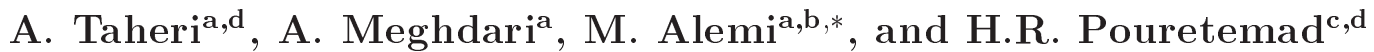 \\ a. Social \& Cognitive Robotics Laboratory, Center of Excellence in Design, Robotics and Automation (CEDRA), Sharif University \\ of Technology, Tehran, Iran. \\ b. Faculty of Humanities, West Tehran Branch, Islamic Azad University, Tehran, Iran. \\ c. Institute for Cognitive and Brain Sciences (ICBS), Shahid Beheshti University, Tehran, Iran. \\ d. Center for the Treatment of Autistic Disorders (CTAD), Tehran, Iran.
}

Received 8 May 2017; accepted 4 December 2017

\section{KEYWORDS \\ Music-based therapy; \\ Xylophone; \\ Autism Spectrum \\ Disorders (ASD); \\ Human-Robot \\ Interaction (HRI); \\ Social robots; \\ Social and cognitive \\ skills; \\ Imitation; \\ Joint attention.}

\begin{abstract}
Utilizing a humanoid social robot to systematically teach music to children with autism has not received enough attention to date. In this study, a novel robotassisted music-based scenario was designed in order to: 1) teach fundamentals of music via a xylophone-/drum-player robot as a teacher assistant, and 2) improve social/cognitive skills through active music games in children with autism. The educational-therapeutic interventions were conducted in an eleven-session case study program on three highfunctioning and one low-functioning children with autism taking into consideration the children's, parents', and therapists' experience during the program. The results indicated that, as a tool and facilitator, the NAO robot does have the ability to teach musical notes/rhythms to the participants with high-functioning autism. It was also observed that the severity of the participants' autism as well as the stress of the parents decreased somewhat during these sessions. Furthermore, noticeable improvements were seen in social/cognitive skills of all four participants as well as in the positive effect of this program on fine motor imitation skills of two subjects after the interventions. The progress reported from this preliminary exploratory study confirmed the potential benefits of using social robots and intelligent technologies as facilitators in music-teaching and cognitiverehabilitation.
\end{abstract}

(C) 2019 Sharif University of Technology. All rights reserved.

\section{Introduction}

Music has the power to influence humans and, in particular, children's emotions, moods, and feelings. Teaching music can help develop new or improve existing social, verbal/non-verbal communication skills in children [1-3]. Children who receive regular music

\footnotetext{
* Corresponding author

E-mail addresses: taheri@mech.sharif.edu (A. Taheri); meghdari@sharif.edu (A. Meghdari); alemi@sharif.edu (M. Alemi); h-pouretemad@sbu.ac.ir (H.R. Pouretemad).
}

doi: $10.24200 /$ sci. 2017.4608 education may have better movement, math, and reading skills in comparison to their peers [4].

Children with autism may have stereotyped behaviors and limited communication skills [5]. Music could be an effective way to involve them in rhythmic/non-verbal communication [1]. Nowadays, at least $12 \%$ of all treatments of ASD (Autism Spectrum Disorders) consist of music-based therapies [6].

Music is used in therapeutic sessions for children with mental and behavioral disabilities [7]. There is ample evidence that shows either playing music during therapy sessions or teaching music to children with autism can significantly increase the impact of therapy sessions $[8,9]$. These studies have inspired researchers 
to use embodied music-based approaches to facilitate multi-system development of children [6]. In such studies or therapy sessions, an instrument is either played by a human or recorded music is played back in individual/group interventions [6,10]. Kalas [11], and Kim, et al. [2] showed improvement in joint attention, turn taking, and eye contact of children with autism in (active) music-making interventions. Some studies have reported an acceptable decrease in stereotyped behaviors and self-injuries in individual with autism after running music-based interventions [12,13]. Music therapy interventions have also been used to increase social [14,15] and emotional [16] skills, verbal/gestural communication [17], and behaviors [18] of participants with ASD. The lack of studies on improving gross and fine motor skills of children with ASD through musicbased interventions is still a serious gap in this area [6].

Alternatively, the use of robotics technology in different aspects of autism research (e.g., trying to improve eye contact, social and cognitive skills of children with ASD, etc.) is increasing [19-22].

This paper intends to combine the potentials of music education/therapy and robotic technology in autism research. To the best of our knowledge, utilizing a humanoid robot to systematically teach music to children with autism has not received enough attention [19,23-25]. Gifford et al. [23] used a drum-player NAO robot to provide consistent repetitive training to teach musical rhythms to typically developing children and individuals with autism; in their preliminary attempt, they reported some potential improvements in intralimb and interlimb coordination, conversation bouts and rates of joint attention bits. Peng et al. [24] programmed a Darwin robot to play a xylophone and involved two children with autism in a seven-week study in imitation games; they reported some progress in the subjects' concentration and communication, yet did not see significant improvement in imitation tasks. In an interesting recent study, Srinivasan et al. [25] discovered the importance of robotic and music-based interventions by running and comparing the effects of rhythm, robotic, and comparison interventions on the social attention patterns of 36 children with autism. Their NAO robot did not play musical instruments; however, its task was to involve the subjects in social and cognitive games, singing, and making requests.

To this end, a novel systematic robot-assisted music-based intervention scenario has been designed to: 1) primarily teach the fundamental concepts of how to play drum and xylophone to children with autism using a NAO robot as a teacher's assistant (facilitator), and 2) affect social and cognitive skills as well as motor imitation of children with autism. It is intended to explore the effect of the interventions on three groups who have been explicitly or implicitly involved in the program: a) participants with autism who receive the music education, b) their parents, and c) the human therapists. The purpose of this study is to find preliminary exploratory answers for the following research questions: a) Does a social robot (as a teacher's assistant) have the ability to teach music (i.e., notes and rhythms) to children with autism?; b) Can robot-assisted interventions affect social and cognitive skills in children with autism through music education?; c) What is the impact of robot-assisted music education intervention on the stress of the subjects' parents during the program?; d) How acceptable is it to have a robot as a co-teacher in music education for the human therapists?

\section{Research methodology}

Study approvals: Ethical approval for the protocol of this study was provided by the Iran University of Medical Sciences (\#IR.IUMS.REC.1395.95301469), and the certification for ABA (Applied Behavioral Analysis) and robot-assisted therapy with autistic children was received from the Center for the Treatment of Autistic Disorders (CTAD), Iran. Our music teacher in this study has dual university degrees: BA in psychology (a specialist in the education of exceptional children and children with special needs), BA in art (music), and also has the certification in application of music in physical and mental health for the children from the Music Application in Mental and Physical Health Association (MAMPHA), Iran based on international music-therapy standards. (While there is no musictherapy majors in Iran's universities, MAMPHA is the pioneer and the valid active organization in the field and provides a wide range of services including consultation, workshops, annual congresses, and actual music therapy.) The parents/children voluntarily took part in the program and signed a pledge/consent form to maintain high moral standards.

\subsection{Participants}

The criteria for volunteers to take part in our program were a) being diagnosed with autism spectrum disorders, b) being at least 3.5 years old, c) having the ability to understand/obey the instructions, d) minimum cognitive development of a 3 -year old, e) being able to do simple imitation movements, and $\mathrm{f}$ ) not having a musical learning background. At first, six families volunteered to take part in this program. Two of them were excluded from the study because: 1) one of the children was scared from the robot at the introduction session and his family refused to bring their 4-year-old low-functioning son to the music program anymore; 2) unfortunately, the 4.5-yearold low-functioning volunteer's parents faced a serious personal problem at the time of the program and did not continue the education after the third intervention 
Table 1. The participants' details.

\begin{tabular}{|c|c|c|c|}
\hline \# & Name & Age & Autism severity \\
\hline 1 & P1-Ben & 6 years and 3 months & High-functioning autism, with hyperactivity; MLU $^{\mathrm{a}}>6$ \\
\hline 2 & P2-Moh & 6 years and 1 month & High-functioning autism; MLU $>6$ \\
\hline 3 & P3-Rad & 6 years and 5 months & High-functioning autism, with verbal deficits; MLU $<6$ \\
\hline 4 & P4-Roh & 6 years and 6 months & $\begin{array}{l}\text { Low-functioning autism, with intellectual disability, } \\
\text { poor verbal skills, and lack of eye-contact; } M L U<2\end{array}$ \\
\hline
\end{tabular}

a: MLU: Mean Length of Utterance; MLU was assessed by the speech therapists of the CTAD.

session. Finally, three children with high-functioning and one child with low-functioning autism enrolled in this research study (Table 1). All of the participants were approximately 6-year-old males (average: 6 years and 3.8 months old; standard deviation: 2.2 months). We refer to the subjects as P1-Ben, P2-Moh, P3$\mathrm{Rad}$, and P4-Roh throughout this paper. In the area of participants' recruitment, $67 \%$ of the volunteers participated in the whole sessions. In the result section, more information as a preliminary estimate of acceptability and feasibility of our trial study design will be presented.

\subsection{Humanoid robot}

The humanoid robot used in this research is the NAO H-21 robot made by the Aldebaran Company [26]. The capabilities of NAO as well as its suitable programming interface make it a commonly used robot for autism research $[25,27]$. We have used the Iranian boy's name, "Nima", for the robot. Unlike its simple robotic face, the capability of changing eyes' color as well as eliciting speech prosody can make it an appropriate tool for communicating with children with ASD.

\subsection{Musical instruments}

Considering that the patient will be more deeply involved when an instrument sound is simple and pleasant [28], we have chosen a drum and a xylophone for the robot to play in our interventions. Selecting the mentioned musical instruments is standard worldwide and is grounded in some psychological theories [28]. Drums are the basic instruments to teach rhythms to children. Moreover, using a xylophone can develop the basic music skills of children and improve their eyehand coordination. Xylophones have visible colorful bars which makes it easier to learn musical notes. In the very beginning stage, learning music through a xylophone is much easier than the other instruments (e.g., piano, guitar, etc.). For children with autism with atypical fine motor skills, using fingers to play the notes is more difficult than using mallets.

\subsection{Technical design of the games}

Two general music games have been designed to involve children in the interventions: a) playing a real drum/xylophone in robot-child or robot-childtherapist/parent imitation turn-taking games and b) playing a Kinect-based virtual xylophone on the screen in child-robot/parent mode.

\subsubsection{Playing the Drum/Xylophone}

NAO robot has been programmed to play the drum/xylophone. We attached mallets to the robot's hands. A configurable user-friendly GUI (Graphical User Interface) (Figure 1(a)) and some rhythm patterns by Choregraphe [26] software (Figure 1(b)) have been

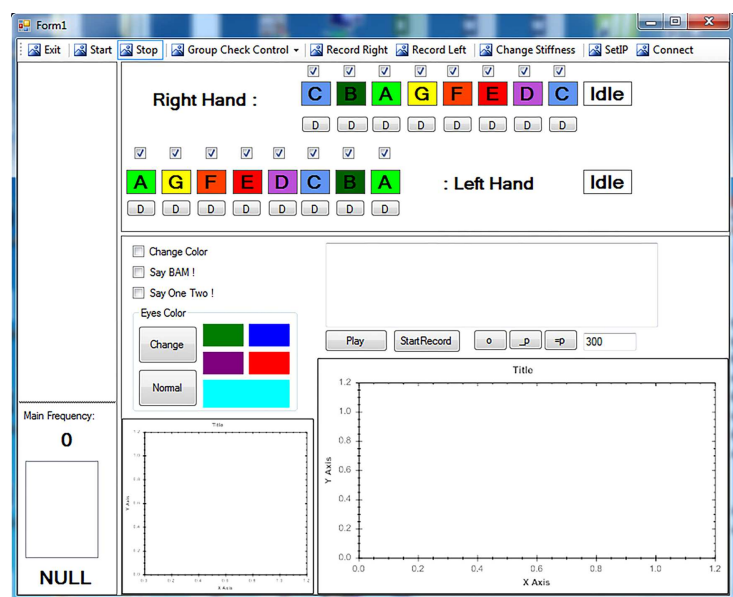

(a)

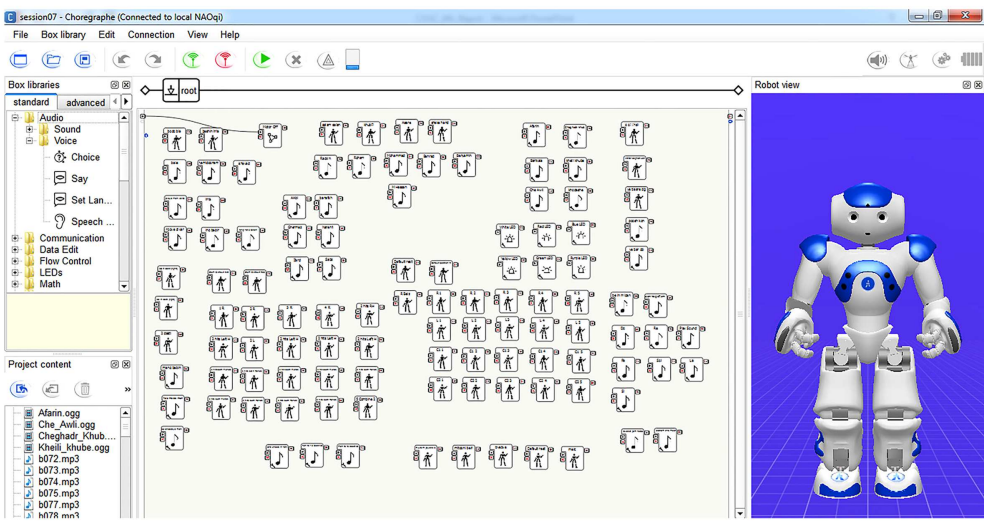

(b)

Figure 1. Software used by the robot-operator during the music-based sessions: (a) Developed GUI and (b) designed rhythm patterns by Choregraphe [26] software. 


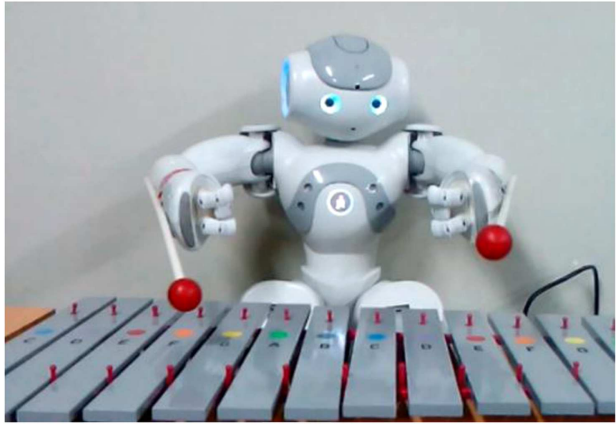

(a)

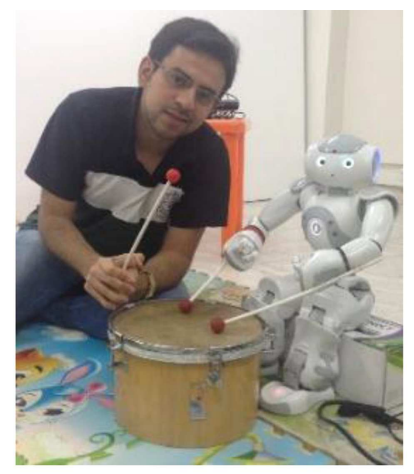

(b)

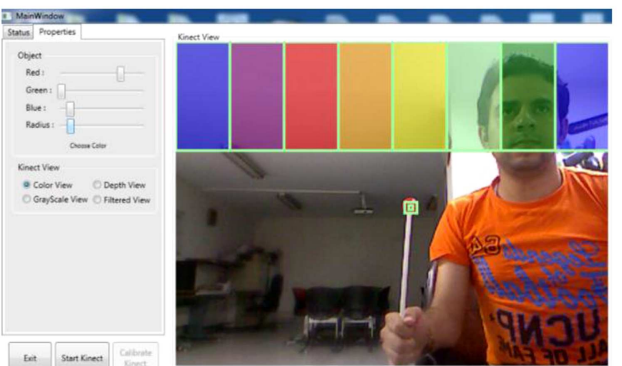

(c)

Figure 2. The Nima Robot, robot operator, and instruments: (a) Real xylophone, (b) drum, and (c) designed virtual xylophone.

developed in order to run different modes of the music scenarios either manually by the operators or automatically in real-time. The robot is able to a) greet, b) play different rhythms and notes with its right/left arm (while directing its attention/gaze to the xylophone's bar and the participant's face), c) change the LED color of its eyes to match the played bar's color and encourage the child to keep eye-contact with the robot, d) play whole/half/quarter notes in melodies and sequences, e) count "1-2" or say "Baam-Baam" while playing, and f) play a sequence of notes given by the operator (Figure 2(a) and (b)).

Audio signals were also captured by the Kinect with $16 \mathrm{kHz}$ data sample rates; after applying Fast Fourier Transform (FFT), the frequency of the note played by the child/therapist/robot was detected and sent to the robot as feedback. Therefore, in its automatic mode, the robot was able to determine if the player's performance was correct and give him/her applause or appropriate hints.

\subsubsection{Virtual xylophone}

A Kinect-based virtual xylophone containing 8 colored bars programmed by C\# WPF has been developed. In this game, the player can see the music bars (with the same color as the real xylophone) as well as himself/herself on the screen. The participant can hit the bars using colored mallets/objects or the palm of their hands. The sounds of one octave from C4 $(261.6 \mathrm{~Hz})$ to $\mathrm{C} 5(523.2 \mathrm{~Hz})$ are heard when hitting these bars. The Toub.Sound.Midi Library was used in order to generate music notes with the computer. Moreover, using the Aforge. Net 2.2.5 Library has enabled us to filter the colors based on RGB and detect the mallets in the images. The coordinates of the mallets' head or palms are also automatically detected by the software (Figure 2(c)).

\subsection{Experimental setup}

The case study design was conducted in the Social \& Cognitive Robotics Lab at Sharif University of Technology with four autistic children in the presence of a human therapist, a humanoid robot, and a robot operator. Time duration of each intervention session was 20-30 minutes. The room size was $10 \times 5 \times 3 \mathrm{~m}^{3}$ and contained "Observation" and "Examination" areas (Figure 3(a) and (b)). The sessions were recorded by three cameras. The child's responses to each activity were also recorded to keep track of his performance. Additionally, two computers, a video projector, and one/two Kinect sensor(s) were also used in the room.

Child-robot interactions were structured and preset. The games had pre-defined purposes and the instructions were described by the robot/therapist before

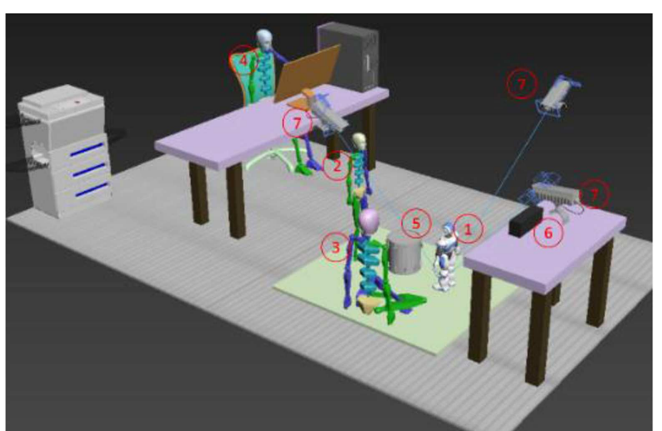

(a)

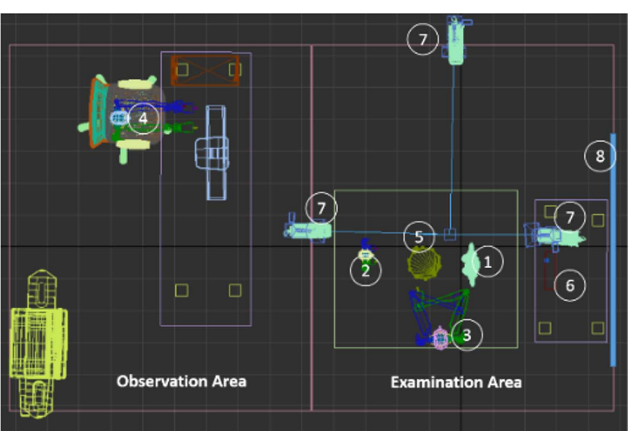

(b)

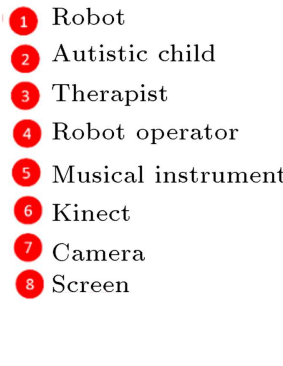

(2) Autistic child
(3) Therapist
(4) Robot operator
5 Musical instrument
6 Kinect
(7) Camera
8 Screen

top view. 


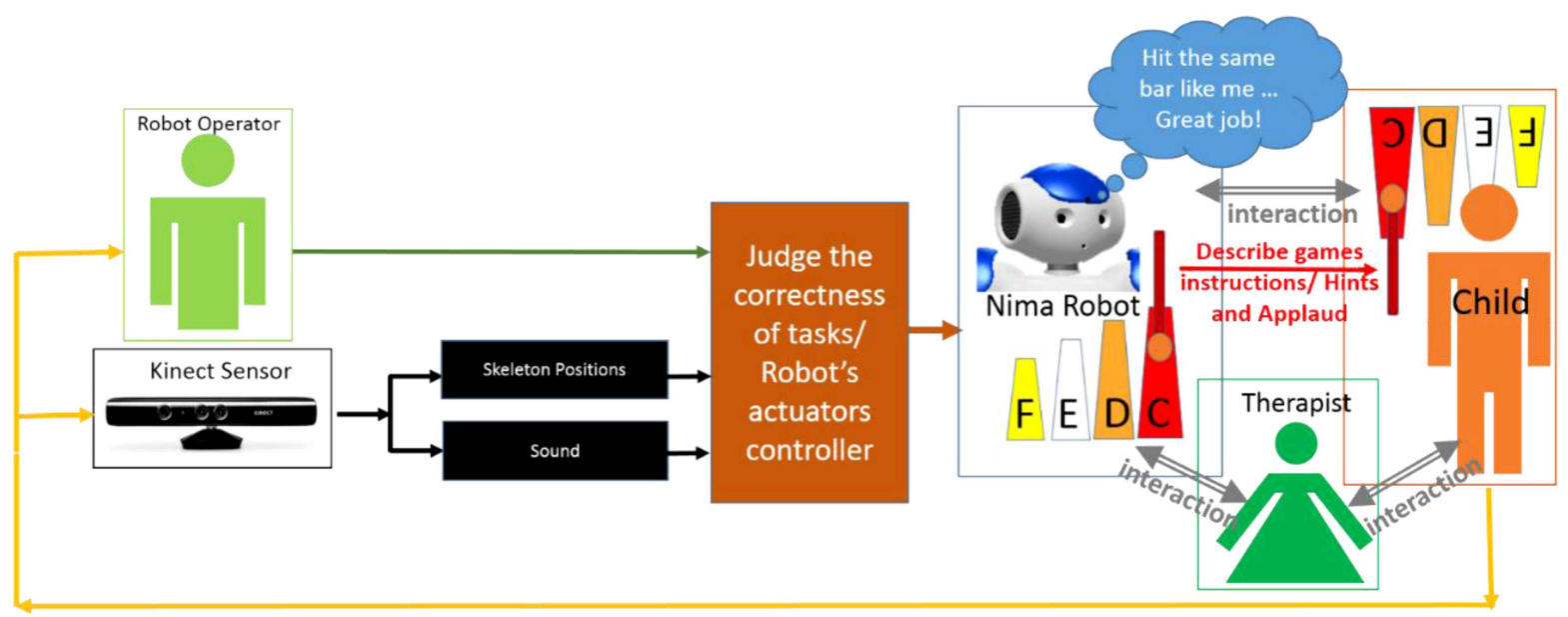

Figure 4. Protocol prompt design.

each game. A Wizard of OZ (WOZ) style robot control has been used, and most of the time, the robot operator sent appropriate real-time voice/motor commands to Nima after seeing the child's performance. Our case study design $[29,30]$ contains two sessions as a baseline without the robot-assisted music teaching. These sessions occurred in week \#1 (including the baselinetest), and week \#2 (including the pre-test just before unveiling the introduction of the robot in the orientation session). Next, nine sessions (i.e., weeks \#3-11) as the teaching interventions were conducted for each child. At the end of the last session (i.e., week \#11), we run the post-test; four weeks later, the follow-up test was conducted with only the questionnaires. Our different assessments all occurred without the presence of the robot. As a case study approach, our focus was on comparing each participant's skill/behavior with his previous behavior based on assessment tools.

\subsection{Intervention protocol}

The purpose of this music-based robot-assisted intervention is a preliminary exploratory attempt to:

a) Teach the fundamentals of music (i.e., musical rhythms and notes);

b) Explore whether this educational-therapeutic protocol could affect positively on:

b.1 A decrease in autistic impairments;

b.2 Improvement/generalization of social and cognitive skills;

b.3 Fine motor imitation skills of the subjects through involving them in active music games based on imitation and joint attention (as two core symptoms of ASD).

Table 2 shows the proposed schedule of the music-based teaching/therapy protocol containing hierarchical and step-by-step activities (extracted from regular/traditional music classes) along with short descriptions/aims of each item. These items are based on active music tasks which pressed the participants to react to the robot in the items such as: 1) playing the drum/xylophone, 2) rhythm perception, 3) working memory games, 4) teaching the notes, 5) involvement in turn-taking group games, 6) reading/playing short music sentences, and finally 7 ) generalizing the learned knowledge to another instrument (i.e., the virtual xylophone). Parents were also asked to be in the room for 5 minutes in the last two sessions and engage in turntaking modes with their children. The participants were involved in dyadic and triadic child-robot and child-robot-therapist/parent interactions. The protocol prompt design is shown in Figure 4.

At each intervention session, each child entered the room and sat in front of the robot and the xylophone/drum (Figure 3(a)). After greetings, based on the previous performance/weakness he showed in the prior sessions and following the music interventions schedule (Table 2), the robot/therapist reviewed the former games with the child and, then, presented new tasks. Appropriate feedback was given to the child immediately by the robot/therapist after watching his performance. Each game (Table 2) contained at least twelve exercises (including different arrangements of the notes, rhythms, and sequence of using hands) while each exercise was presented at most four times. A success rate of $50 \%$ was the defined criterion to go to the next (and most likely higher level) game.

\subsection{Assessment tools}

To find preliminary answers for the research questions of this study, three types of measuring instruments, i.e., human assessments, questionnaires, and interviews, were used at different times:

a) The following Human assessments were conducted by two clinical child psychologists (an autism specialist and a music teacher): 
Table 2. The designed music-based therapy interventions schedule.

\begin{tabular}{|c|c|c|c|c|}
\hline Week\# & Outline & & Games' modes and descriptions & Purpose of the game \\
\hline 2 & Orientation session & & $\begin{array}{l}\text { After conducting the Pre-test in week two, } \\
\text { Nima robot was introduced to the child, } \\
\text { showing robot's greeting, singing, dancing, etc. }\end{array}$ & $\begin{array}{l}\text { Familiarize the child with the } \\
\text { robot/class' environment }\end{array}$ \\
\hline 3 & $\begin{array}{l}\text { Strike the drum } \\
\text { and/or xylophone } \\
\text { bars using mallets }\end{array}$ & $3 \mathrm{~d}$ & $\begin{array}{l}\text { No bars were removed from the xylophone. } \\
\text { Child should hit an arbitrary bar } \\
\text { after each robot's strike. } \\
\text { Some of the bars are removed. } \\
\text { Repeat } 3 \text { a with fewer bars on the xylophone. } \\
\text { Repeat 3a: Child should use both of } \\
\text { hands in order to play the instruments. } \\
\text { Repeat } 3 \text { b: Child should use both of } \\
\text { hands in order to play the instruments. }\end{array}$ & $\begin{array}{l}\text {-Improve fine motor skills } \\
\text {-Visual pursuit } \\
\text {-Learn how to make sounds } \\
\text { with the instruments } \\
\text {-Joint attention }\end{array}$ \\
\hline 4 & Matching the colors & $4 \mathrm{a}$ & $\begin{array}{l}\text { Child imitates the robot's strike } \\
\text { considering bars' color (preferable colors: } \\
\text { red, blue and yellow). } \\
\text { The robot strikes a bar and child points } \\
\text { to the same color on the board/screen } \\
\text { (and vice versa). } \\
\text { The robot's eye LED color changes } \\
\text { and child has to hit the same color } \\
\text { on the xylophone. }\end{array}$ & $\begin{array}{l}\text {-Colors' recognition } \\
\text {-Joint attention } \\
\text {-Instruction perception }\end{array}$ \\
\hline 5 & $\begin{array}{l}\text { Hitting bars } \\
\text { while counting }\end{array}$ & $5 \mathrm{~b}$ & $\begin{array}{l}\text { Imitating the robot's strikes considering } \\
\text { the number of played notes in order. } \\
\text { The child/robot should count } \\
\text { "one-two" while hitting the bars. } \\
\text { Repeat 5a while saying } \\
\text { "Baam-Baam" }\end{array}$ & $\begin{array}{l}\text {-Improve verbal short } \\
\text {-Term memory } \\
\text {-Numbers' perception } \\
\text { and counting }\end{array}$ \\
\hline 6 & $\begin{array}{l}\text {-Hitting bars } \\
\text { without counting } \\
\text {-Dancing }\end{array}$ & $6 \mathrm{a}$ & $\begin{array}{l}\text { Imitating robot's strikes without counting. } \\
\text { Imitating robot's singing / dancing in time break. }\end{array}$ & $\begin{array}{l}\text {-Auditory discrimination } \\
\text {-Making the games' } \\
\text { instructions more complex } \\
\text {-Improve motor imitation }\end{array}$ \\
\hline 7 & Rhythm perception & & $\begin{array}{l}\text { Imitating the robot striking } 3 \text { or } 4 \text { notes } \\
\text { in order while considering the time interval } \\
\text { between each note (on both the drum } \\
\text { and the xylophone). }\end{array}$ & $\begin{array}{l}\text {-Auditory imitation skill } \\
\text {-Hand-eye coordination } \\
\text {-Visual pursuit }\end{array}$ \\
\hline 8 & $\begin{array}{l}\text { Working } \\
\text { memory game }\end{array}$ & & $\begin{array}{l}\text { The robot shows/says two or more colors } \\
\text { to the child, and he/she has to remember } \\
\text { and hit xylophone bars } \\
\text { with the same color. }\end{array}$ & $\begin{array}{l}\text {-Working memory and } \\
\text { dual task performance } \\
\text {-Joint attention }\end{array}$ \\
\hline 9 & $\begin{array}{l}\text { Teaching and } \\
\text { reading the notes }\end{array}$ & & $\begin{array}{l}\text { Cards/labels with the same colors } \\
\text { as the bars are shown to the child; } \\
\text { he/she has to repeat the note's name } \\
\text { and strike the same note on the xylophone. }\end{array}$ & $\begin{array}{l}\text {-Shifting attention skill } \\
\text {-Auditory memory } \\
\text {-Teach the music notes }\end{array}$ \\
\hline
\end{tabular}


Table 2. The designed music-based therapy interventions schedule (continued).

\begin{tabular}{|c|c|c|c|c|}
\hline Week\# & Outline & & Games' modes and descriptions & Purpose of the game \\
\hline 10 & $\begin{array}{l}\text { Playing music } \\
\text { sentences }\end{array}$ & & $\begin{array}{l}\text { Child plays short phrases of the notes } \\
\text { (i.e., a music sentence) while saying } \\
\text { each note's name and the robot assesses } \\
\text { his/her performance, i.e., playing easy } \\
\text { and preliminary songs (parents can also } \\
\text { attend the class and observe the last } \\
\text { performance of their children). }\end{array}$ & $\begin{array}{l}\text {-Cooperation between behavior } \\
\text { and motor skills } \\
\text {-Auditory memory } \\
\text {-Play music sentences in the } \\
\text { presence of the parent }\end{array}$ \\
\hline 11 & $\begin{array}{l}\text {-Playing the virtual } \\
\text { xylophone } \\
\text {-Involving children } \\
\text { in turn-taking games }\end{array}$ & $11 b$ & $\begin{array}{l}\text { The robot hits some of the xylophone bars } \\
\text { and expects the child to imitate it on the } \\
\text { virtual xylophone (and vice versa). } \\
\text { A game to involve the parents in the } \\
\text { last session: The therapist/parent hits } \\
\text { the real/virtual bars and expects the } \\
\text { child/robot to imitate on the virtual/real } \\
\text { xylophone, and vice versa. } \\
\text { (expecting the child to generalize the } \\
\text { knowledge on the virtual xylophone). }\end{array}$ & $\begin{array}{l}\text {-Sharing attention between } \\
\text { different tasks } \\
\text {-Improve turn-taking } \\
\text {-Involve the child in } \\
\text { child-robot-mother/ } \\
\text { therapist group games } \\
\text {-Generalization skills }\end{array}$ \\
\hline
\end{tabular}

a1) Colors'identification/expression test was implemented in order to assess the subjects' ability of recognizing different colors;

a2) To investigate music learning ability: 1) Stambak's Rhythmic Structures Reproduction test [31], which contains 21 (easy to hard level) rhythmic tasks in which the participant should reproduce the patterns on a drum after hearing (and not seeing) them was conducted. The subjects have at most two chances to successfully complete each of test's item; 2) A short music sentence was given to the children, and the participants were asked to play it while using their left/right hands in order as well as saying the notes' name;

a3) Assessment of Socio-Cognitive (focusing on imitation and joint attention) skills of each participant was conducted using 30 items including a) 15 designed fine/gross motor imitation tasks and b) extracting 15 items from the Early Social Communication Scales (ESCS) test $[5,32]$. ESCS is a comprehensive well-known clinical measure of joint attention behaviors, behavioral requests, and social interaction behaviors of children. During the socio-cognitive assessment, different tasks and objects were presented to the participant to observe his/her initiating/responding to social and communicative behaviors with the therapist. b) Three valid questionnaires in autism research. All of the questionnaires were filled in by the parents four times.

b1) Gilliam Autism Rating Scale (GARS) [33] is a questionnaire for estimating autism severity, covering subscales: Stereotyped behaviors, communication, social interactions, and developmental disturbances;

b2) Autism Social Skills Profile (ASSP) [34], is a comprehensive tool to measure social behaviors of an individual with autism from 6 to 17 years old. ASSP consists of 45 items. Three subscales, i.e., social interaction, social reciprocity, and detrimental social behaviors, can be measured with this questionnaire;

b3) Parenting Stress Index-Short Form (PSI-SF) [35] is a tool to measure parenting stress.

Due to the small number of the participants in this study, no scientific statistical analysis (i.e., significant tests) can be run on the data; however, the Cohen's d effect size [36] is calculated among the pre-test and post-test results of the questionnaires, each in two situations: a) considering all the four participants as a group and b) excluding the lowfunctioning subject and considering the three highfunctioning subjects. The Cohen's d effect size is considered, which is independent of the sample size, as an estimation measure of the treatment effect. It has been used not for making strong claims and generalizing the observed results, but for finding the 
potentials of the robot-assisted interventions' effect on children in active music sessions (Table 5).

c) Interviews with the parents and the therapists before and after the program:

c1) Interview with the parents to be informed of any children's behavioral changes during/after the interventions in their real life;

c2) Interview with the therapists to find their opinion/experience about having a robot as a co-teacher in music sessions.

\section{Results and discussion}

After two non-robot baseline sessions and conducting the baseline-test and pre-test in weeks \#1 and \#2, the Nima robot was introduced to the participants in the second half of week \#2; in addition, the verbal and movement capabilities of the robot were shown in the presence of each subject, his parents, and the teacher. The purpose of this session was to familiarize the participants with the class environment as well as observe the child's tendency to start/keep communication with Nima. Music was not taught in the first two weeks. After that and based on the designed protocol presented in Subsection 2.6, music was taught stepby-step by the robot/teacher during the rest of the sessions (Figure $5(\mathrm{a})-(\mathrm{d}))$. We were able to run all of the games (i.e., games \#3a to \#11b) for the three high-functioning subjects during the intervention sessions; however, for the low-functioning P4-Roh, games \#8a-\#11b were not systematically played because of his serious deficits in cognitive skills and instruction perception. Therefore, in the area of delivery of tasks' conditions' feasibility, acceptable performance of the high-functioning subjects was observed. The post-test was run at the end of week eleven for all the assessment tools. In this section, the observed results of our assessment tools will be presented case by case.

\subsection{Colors' identification and expression test} In this study, "identification" and "expression" of colors were assessed for the autistic participants three times.

- P1-Ben and P2-Moh: They had no problem in identifying, expressing, and generalizing the colors.

- P3-Rad: For P3-Rad, color "identification" was better than color "expression" in both the baselinetest and the pre-test. His main difficulties were in identifying/expressing pink, yellow, and purple colors. P3-Rad could accurately find the colors in the background without too many colors. On the pretest, we observed that P3-Rad had a serious problem with "generalizing" the colors as well as "instruction perception". Questions about the colors were asked

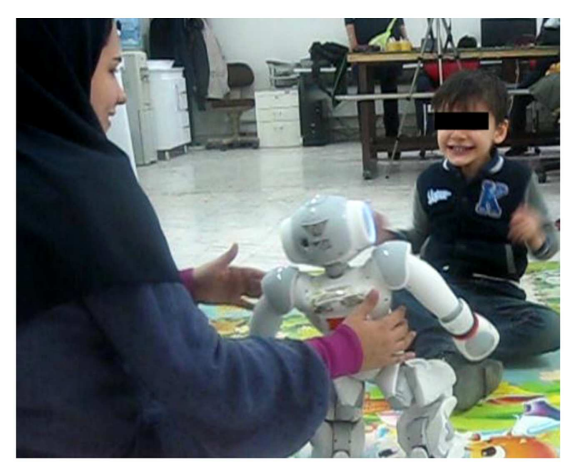

(a)

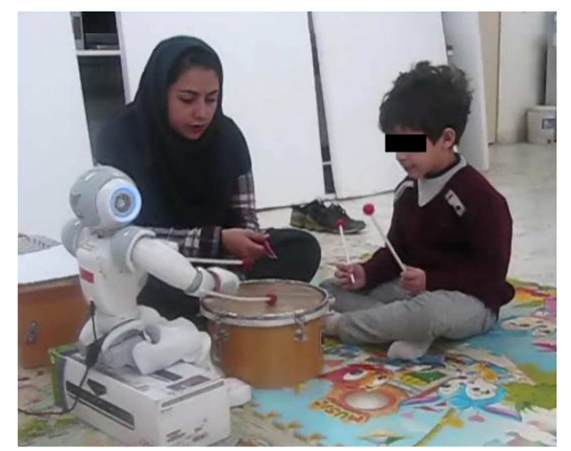

(c)

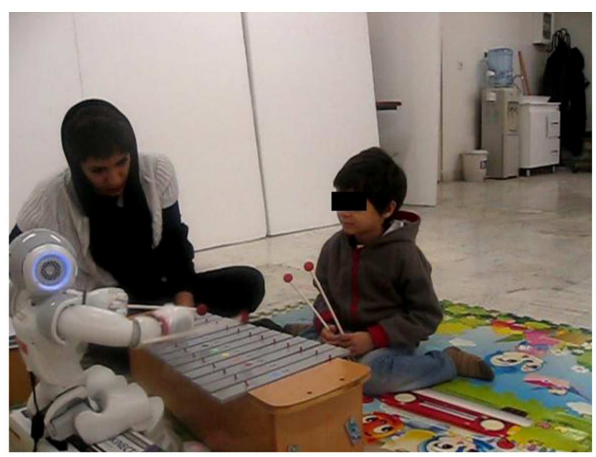

(b)

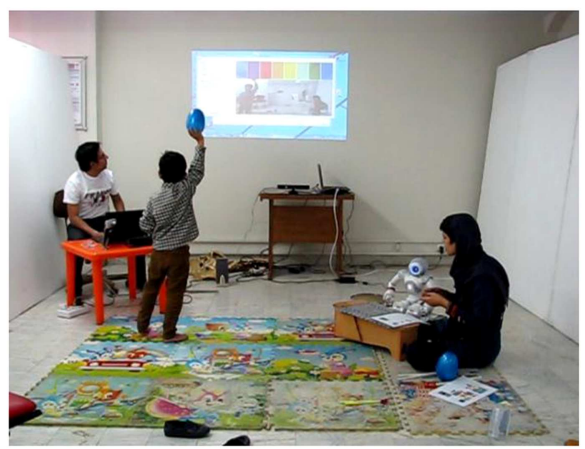

(d)

Figure 5. Snapshots of the interventions: (a) P4-Roh in the orientation session, (b) P2-Moh is imitating the robot (game \#4a), (c) P1-Ben is doing a rhythm perception task (game \#7a), and (d) P3-Rad is imitating the robot (game \#11a). 


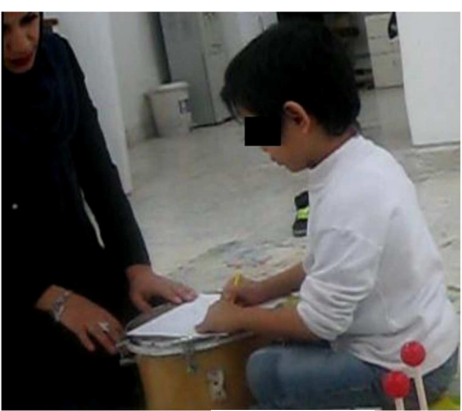

(a)

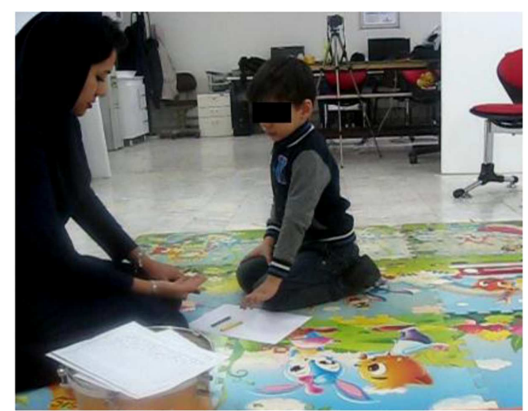

(b)

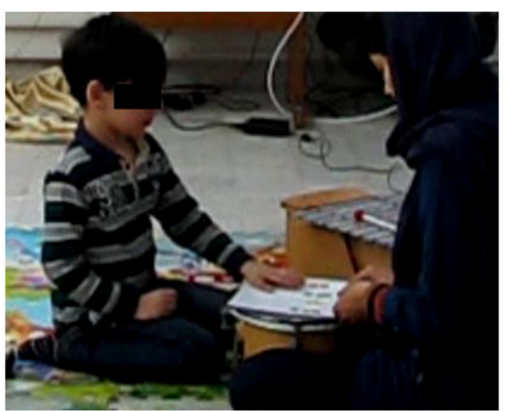

(c)

Figure 6. Color identification/expression test: (a) P3-Rad is drawing (instead of coloring) a fish with yellow pencil in the pre-test, (b) P4-Roh could not identify the blue color in a background with two colors in the baseline-test, and (c) P4-Roh could successfully classify 5 different colors in the post-test.

in a different way in the pre-test, as compared to those in the baseline-test. Some objects, like a fish, sun, and ball, were sketched, and he was asked to "color the pictures with a yellow pencil"; however, P3-Rad took the pencil and drew another fish (Figure 6(a)). Fortunately, in the post-test, his problem with the "expression" of yellow and purple colors was resolved, and he was able to identify/express the colors "used for the xylophone bars". However, this deficit slowed down his music learning process slightly.

- P4-Roh: P4-Roh did not have the ability to identify (and obviously express) any colors at all in the baseline-test and pre-test. He could not even identify a color in the background with only two existing colors (Figure 6(b)). However, the noteworthy changes observed for P4-Roh in the post-test include the expression of the "blue" color as well as the ability to separate/classify colors in the background with five different colors (Figure 6(c)). It seemed that the colors' "identification" process started occurring for P4-Roh. His frequent/continuous involvement in color-based games, such as $4 \mathrm{a}, 4 \mathrm{~b}$, and $4 \mathrm{c}$ (Table 2 ), during three months could be the reason for this observation.

\subsubsection{Subsection's summary}

The observations of P3-Rad and P4-Roh's performance show the potential of our designed scenario to provide improvement in color recognition. In [28], the possibility of color identification through non-robotic teaching music instruments to exceptional children is also addressed.

\subsection{Music learning ability: (a) the Stambak rhythm reproduction test and (b) playing a music sentence.}

In this sub-section, the music learning ability of the subjects is analyzed. The Stambak Rhythm Reproduction Test was run on the autistic subjects three times to investigate each participant's performance in rhythm perception (Figure $7(\mathrm{a})$ ). The results of this test are presented in Table 3. Moreover, in the Posttest, each high-functioning subject was given a short music sentence task (consisting of 12 colored notes on a sheet), and they were asked to read the notes loudly first and, then, play the whole sentence on the xylophone using their right/left hands in order (Figure 7(b)).

- P1-Ben: P1-Ben was a genius in rhythm perception who reached exercise \#18 in the Baseline and

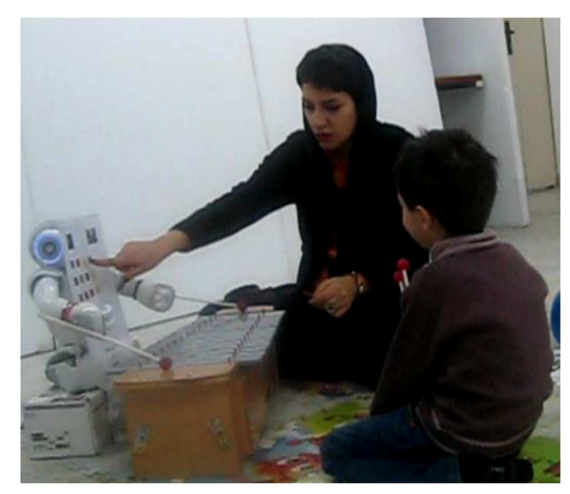

(b)

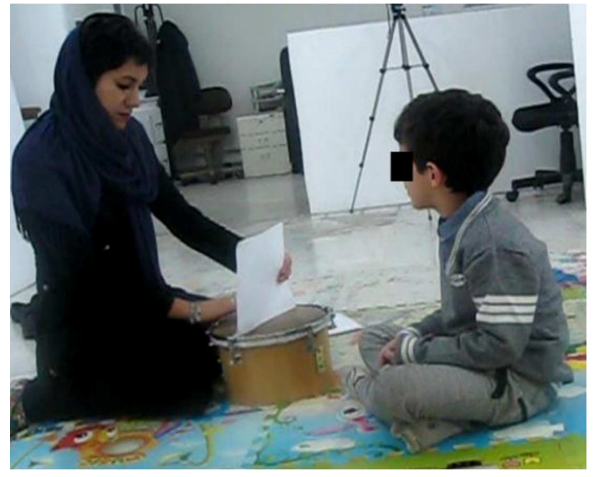

(a)

Figure 7. Assessment of music learning ability: (a) Stambak's rhythm test and (b) saying/playing short music sentences test. 
Table 3. Stambak's rhythm test results in the baseline-test, pre-test, and post-test.

\begin{tabular}{cclll}
\hline \multirow{2}{*}{ \# } & Participant & \multicolumn{2}{c}{ Scores of the Stambak's Rhythm test } \\
\cline { 3 - 5 } & \multicolumn{1}{c}{ Baseline-test } & \multicolumn{1}{c}{ Pre-test } & \multicolumn{1}{c}{ Post-test } \\
\hline 1 & P1-Ben & 18 (in the second try) & 18 (in the second try) & 19 (in the second try) \\
2 & P2-Moh & 6 (in the second try) & 6 & 13 \\
3 & P3-Rad & 10 & 11 (in the second try) & 14 (in the second try) \\
4 & P4-Roh & 0 (did not understand) & 0 (did not understand) & 4 (with the guide of counting) \\
\hline
\end{tabular}

pre-test without any previous music background. However, he stopped at exercise \#19 and could not progress to tasks \#20 or \#21. During the first sessions, P1-Ben usually used only his dominant hand or made many mistakes in choosing the sequence of his playing hands. Although he was able to correctly read the final sentence test, he made three mistakes in changing the playing hands (which was, however, an acceptable performance). The psychologists also reported some progress in his fine hands imitation. The music learning process occurred for P1-Ben.

- P2-Moh: P2-Moh who only reached task \#6 showed the most changes in the rhythmic test and was able to eventually successfully play task \#13 of the Stabmak's test. In playing the sentence task, he concentrated intently on the notes and his playing hands in order not to make any mistakes. He did a great job; howbeit, he was somewhat slower than what we expected. Based on our experience in regular music classes, his performance seemed to be similar to typically developing children. In addition, he showed some improvement in fine motor imitation by being able to use both hands consecutively and spontaneously in playing the instruments during the last three sessions.

- P3-Rad: In the post-test, P3-Rad successfully played task \#14 for the first time. In comparison to the two other high-functioning children, his weakness in expressing/identifying some colors slowed down his music learning process a bit, especially in saying/playing the notes' test and the music sentence games (i.e., games \#9a and \#10a).

- P4-Roh: Our low-functioning subject, P4-Roh, was initially unable to understand any of the rhythm tasks and could only play 2-hits with the therapist's direct verbal guide of "Baam-Baam" (on his fourth try) in the Baseline and pre-test; however, in the post-test, he was able to understand/play tasks \#1 through \#4, each on his second try with direct help of counting "1-2" / "Baam-Baam". He showed no significant improvement in "music learning" during the course of the program. The current proposed music schedule is too cumbersome for our low-functioning subject. One possible way to make future works effective would be to expand the number of sessions at least by two times for this kind of children. He often played randomly on the xylophone bars instead of correctly imitating the robot during the intervention games. The teacher and Nima helped him understand the tasks better (instruction perception). Using both of the hands consecutively to play the drum/xylophone was our ultimate goal for him, and we assumed he would not achieve this skill. While P4-Roh could not imitate the same bars from the robot even at week eleven, he was, however, able to acceptably imitate the switching hands in the last four sessions.

\subsubsection{Subsection's summary}

The three high-functioning participants of this study showed some improvement in playing rhythms as well as being able to read/play simple music sentence test, meaning that the initial music learning occurred for these subjects and the results for the three months of interventions were promising. Improvement in rhythm perception of children could possibly set the body's internal rhythm; as a result, it could positively affect their walking, speaking, and even handwriting [37]. Now, we can answer the first research question as follows: social robots (as teacher assistants) do have the ability to teach fundamentals of music to children with high-functioning autism in robot-assisted programs; however, for subjects with low-functioning autism, no conclusion can still be extracted through this work and more studies need to be done. Moreover, Srinivasan et al. [25] reported progress in fine motor imitation of autistic children during music-based robotic-assisted interventions, which supports the observations of our program for P1-Ben and P2-Moh.

\subsection{Socio-cognitive skills and qualitative behavioral analysis}

To assess the social and cognitive skills of the participants, our psychologist ran thirty items in the pre-test and post-test to evaluate instruction perception, turntaking, social participation, eye-gaze shifting, joint attention, and social imitation skills of the autistic subjects (Figure 8(a)-(f)). Each item was run 2 or 3 times, and the criterion for passing the item was successful performance of 2 times (Table 4). Then, the summarized results of the tests as well as the qualitative summary of the interventions reported by 


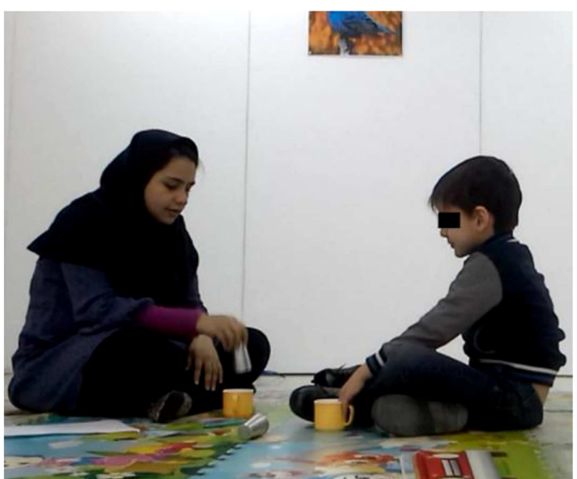

(a)

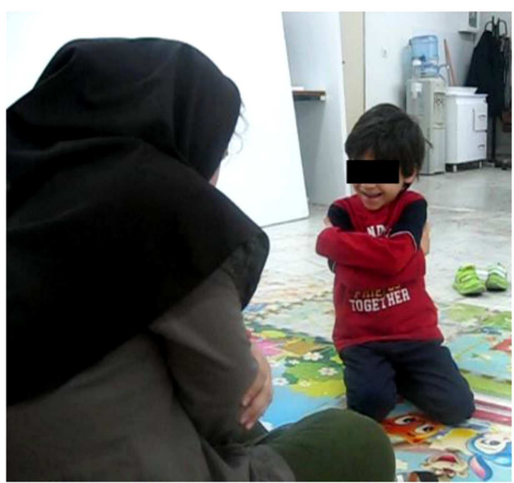

(d)

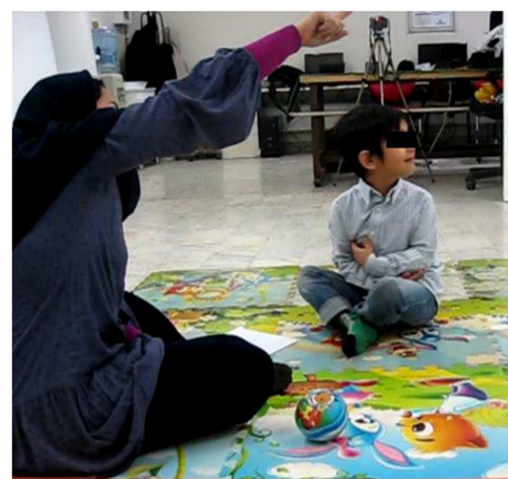

(b)

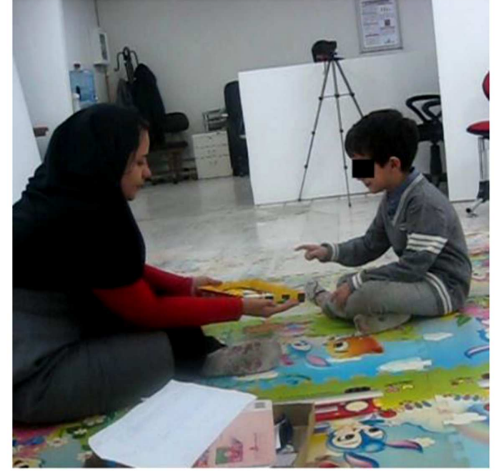

(e)

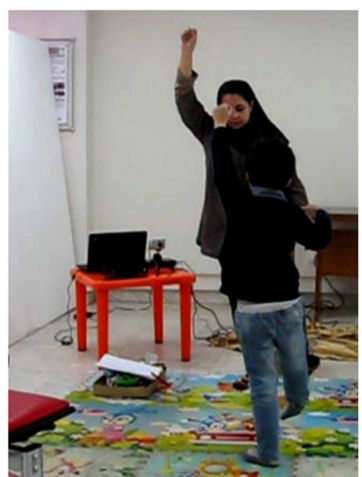

(c)

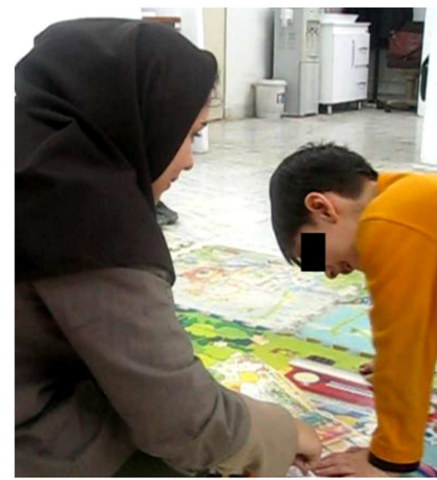

(f)

Figure 8. Assessment of joint attention and imitation skills of the participants by the psychologist: (a) P4-Roh could not successfully imitate the therapist in the game of arranging the objects in the pre-test, (b) P3-Rad responded to joint attention of the therapist in the pre-test, (c) while previously unable to pass this item, P1-Ben successfully did $2 / 3$ of opposite arm-leg imitation game in the post-test, (d) P2-Moh responded to the therapist's poem emotionally by showing the cold feeling spontaneously in the post-test, (e) P1-Ben tried to complete the puzzle by pointing/choosing appropriate elements in the pre-test, and (f) unable to respond to joint attention in the book game in the pre-test, P4-Roh was able to successfully complete this test in the post-test.

Table 4. The scores of socio-cognitive items in the pre- /post-tests (passing criteria is a score greater than or equal to $2 / 3$ ).

\begin{tabular}{|c|c|c|c|c|c|c|c|c|c|c|c|c|}
\hline \multirow[t]{2}{*}{$\#$} & \multirow[t]{2}{*}{ Name } & \multicolumn{5}{|c|}{ Pre-test scores (out of 30 ) } & \multicolumn{5}{|c|}{ Post-test scores (out of 30 ) } & \multirow{2}{*}{$\begin{array}{c}\text { Main obtained } \\
\text { socio-cognitive improvement }\end{array}$} \\
\hline & & $0 / 3$ & $1 / 3$ & $2 / 3$ & $2 / 2$ & Total & $0 / 3$ & $1 / 3$ & $2 / 3$ & $2 / 2$ & Total & \\
\hline 1 & P1- Ben & 3 & 6 & 2 & 19 & 21 & 2 & 4 & 5 & 19 & 24 & Fine motor imitation \\
\hline 2 & P2- Moh & 3 & 8 & 2 & 17 & 19 & 1 & 7 & 4 & 18 & 22 & Fine motor imitation \\
\hline 3 & P3- Rad & 0 & 2 & 3 & 25 & 28 & 0 & 1 & 2 & 27 & 29 & Triad memory \\
\hline 4 & P4- Roh & 13 & 3 & 4 & 10 & 14 & 11 & 3 & 6 & 10 & 16 & Joint attention; instruction perception \\
\hline
\end{tabular}

the clinical psychologists are presented. However, the quantitative/qualitative content analyses of the video records (i.e., the video coding) of this program are not presented here.

- P1-Ben: P1-Ben showed some improvement in fine movement and difficult imitations (e.g., matching the ribbons/matches game and opposing arm-leg imitation game) from the pre-test to post-test. During the interventions, $\mathrm{P} 1-\mathrm{Ben}$ was very motivated to behave maturely in the presence of Nima as he did not want to ruin his friendship with the robot with inappropriate behavior. This observation is similar to the results presented in improving behaviors in $[16,18]$.

- P2-Moh: P2-Moh successfully passed three items in the post-test, which he previously could not do. These items included blowing a balloon, responding physically/emotionally to a poem, and matching ribbons/matches. Although $\mathrm{P} 2-\mathrm{Moh}$ is very resistant to education classes in his real life (mentioned in his medical reports), he did not show any maladaptive behaviors in our course. Moreover, based on the 
psychologists' report, the child felt secure/relaxed during the sessions. Similar to this observation, Finnigan and Starr [38] and Geretsegger et al. [1] also reported the necessity of a non-threatening environment in (non-robotic) music therapy sessions for the participants with ASD.

- P3-Rad: He had the least amount of problem in passing the items. His triad memory, however, improved somewhat, compared with his past, by successfully passing the working memory imitation game in the post-test.

- P4-Roh: P4-Roh was our most interesting case. In the post-test, he showed progress in two joint attention items including pointing to the pictures on the back wall and gaze-shifting in the book game. This observation is in line with the improvement possibility of joint attention skills reported in a non-robotic music therapy study done by Kalas [11], and Kim et al. [2], as well as some roboticassisted interventions research [21]. It should be noted that, following the notes played with eyes, eye-motor coordination movements and gaze-shifting from the robot to the instruments (and vice versa) are some examples of the joint attention situations provided for the subjects in this study. His failure in the items (especially in the pre-test) was due to his serious problem with "instruction perception" as well as his "low attention span". P3-Roham was a calm person with poor verbal and social communication. Based on his mother's report, P4Roh usually refuses social contact with others in the early stages of communication; however, in our music classes, he communicated with two tools: the robot and the musical instrument; therefore, music was the interface between the child and the robot and, in this way, a foundation was built for a successful dyadic child-robot communication. He understood none of the instructions at the beginning of the music program. Slight improvement in his instruction perception, attention, and understanding what happened in the class was a positive note for P4-Roh over time. P4-Roh's attention span also increased somewhat, and he spent more time doing the music tasks. He participated in the class eagerly. Nima was a noticeable help as a communication tool; it raised the child's excitement level and motivated him to take part in the sessions effectively. As one of the interesting potential effects of the current robot-assisted interventions for our low-functioning subject and according to the psychologists' observations, P4-Roh's verbal skills increased somewhat and his stereotyped behaviors (especially his fluttering fingers) decreased a bit. Music seemed to be effective in decreasing the stereotyped behaviors of children with autism $[12,13]$.
Moreover, this is in line with [27,39] who reported some decrease in repetitive/stereotyped behaviors of low-functioning children with autism after running robot-assisted imitation and joint attention games. In fact, engaging in stereotyped behaviors prevents the child from paying attention to surrounding events [5]. Therefore, attempt was made to not allow P4-Roh to remain solitary during the sessions.

\subsubsection{Subsection's summary}

In the human assessment sections (i.e., Subsections 3.1 to 3.3 ), we found that our music sessions represented a non-threating and indirect method for communication, which provided special emotional pleasure/satisfaction for the children. Furthermore, playing the xylophone also required the use of both hands and fingers (i.e., the small muscles/joints). During the sessions, the autistic children had to communicate with the human therapist/parent, the robot, and music tools. Based on the video records and psychologists' report, one of the promising observations for the highfunctioning subjects' performance was their high interest in starting/continuing their music class. Our observation showed that Nima was effective in this regard, and the children's following, curiosity, and questioning increased somewhat in the presence of the robot. The children used many opportunities to push Nima to speak and ask him questions. Similar to our observations, increasing of ASD's communication and feeling of closeness with/to the assistive robots during the interventions were also reported in $[19,21,27,40]$.

\subsection{Questionnaires \\ 3.4.1. GARS}

The GARS questionnaire was filled in by the parents four separate times (Figure 9). A decreasing trend in the autism severity of the participants from pre-test to post-test was observed. The decreasing amount in autism severity, however, seemed to be different and was much higher for the high-functioning participants than that for the low-functioning subject. A detailed subscales' assessment of the GARS questionnaires showed us some improvement in three subscales: "stereotyped behaviors", "communication", and "social interaction" for two of the participants, P1-Ben and P4-Roh, from pre-test to post-test. Based on the GARS, more changes occurred in the "communication" subscale for P1-Ben and in the "social interaction" subscale for P4-Roh, as compared to the other subscales. P3-Rad progressed somewhat in "stereotyped behaviors" and "communication" subscales, while P2-Moh showed some improvement in "communication" and "social interaction". An interesting point of the GARS results, which may not have happened accidently, is the observation of progress for all four subjects in the 


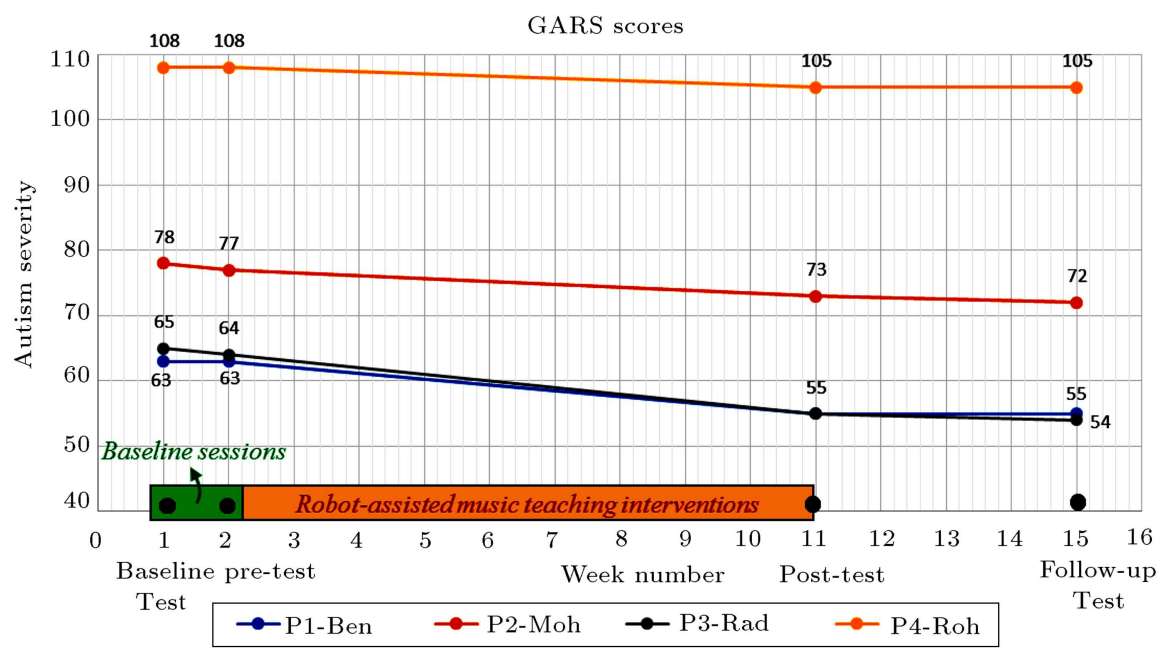

Figure 9. GARS scores (autism severity) of the participants in the baseline-test, pre-test, post-test, and follow-up Test.

Table 5. Cohen's d effect size measurement between pre-test and post-test of the questionnaires' results (the numbers that represent the large or close to being large effect size (i.e. $\sim$ or $>0.8$ ) are in bold in the table).

\begin{tabular}{|c|c|c|c|}
\hline \multirow[t]{2}{*}{ Questionnaire } & \multirow[t]{2}{*}{ Subscale } & \multicolumn{2}{|c|}{$\begin{array}{c}\text { Cohen's d effect size between } \\
\text { pre- and post-test scores }\end{array}$} \\
\hline & & All 4 participants & 3 high functioning children \\
\hline \multirow{4}{*}{ GARS } & Overall scores & 0.269 & 0.762 \\
\hline & Stereotyped behaviors & 0.199 & 1.443 \\
\hline & Communication & 0.212 & 1.231 \\
\hline & Social interaction & 0.338 & 0.362 \\
\hline \multirow{4}{*}{ ASSP } & Overall scores & 0.222 & 0.473 \\
\hline & Social reciprocity & 0.157 & 0.251 \\
\hline & Social participation/avoidance & 0.394 & 1.233 \\
\hline & Detrimental social behaviors & 0.167 & 0.715 \\
\hline PSI-SF & Overall scores & 0.413 & 0.469 \\
\hline
\end{tabular}

subscale "communication" (in their real life). This observation is in line with the therapists' reports of the communication toward the robot/instruments and the possible reasons discussed in Subsections 3.2 and 3.3. According to Table 5, considering the highfunctioning subjects in a group, the calculated effect size of the overall scores of the GARS as well as two subscales, communication and stereotyped behaviors, are large or close to being large, which could show that the interventions had a promising effect on the participants' socio-cognitive skills and, as a result, their autism severity. It is a signal that the presence of Nima, as an attractive communication tool, could be one of the positive keys affecting the communication skills of the children even in their life outside the study. Moreover, a comparison of the GARS overall scores between the post-test and follow-up Test shows the retention and stability of the sessions' impact on each of our subjects.

\subsection{2. $A S S P$}

The results of the ASSP questionnaire are presented in Figure 10. Based on the parents' reports, the social skills of all the participants improved somewhat during the intervention sessions. This observed result is in line with the reports of $[2,6,15,17]$ in non-robotic music therapy programs as well as $[21,41]$ in robotassisted therapeutic interventions. Human therapists' reports in Subsections 3.2 and 3.3 also support the parents' viewpoint in improvement of the subjects' social skills. A detailed overview of the ASSP shows us that scores of the subscale as follows: a) "social reciprocity" increased somewhat for all the participants (changes' mean: 4.25, changes' standard deviation: $1.26)$; b) "social participation/avoidance" increased with the least change reported for P3-Rad (changes' mean: 4.50, changes' standard deviation: 1.91), with a large effect size (Table 5) for the high-functioning children group; and c) "detrimental social behaviors" 


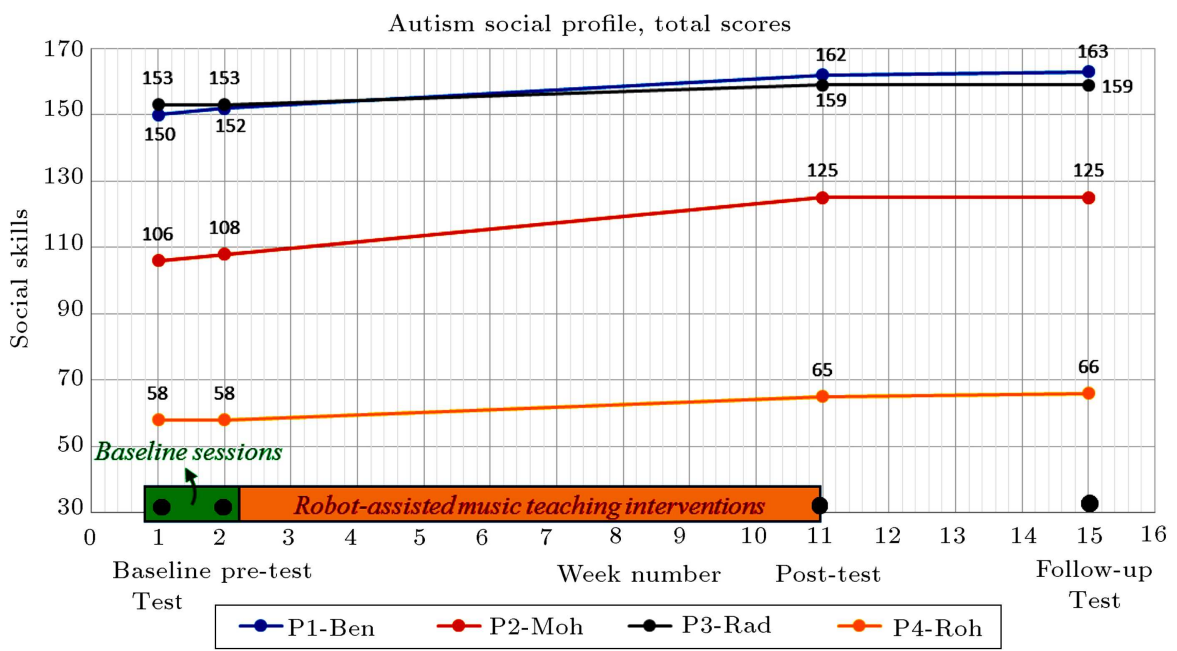

Figure 10. Total ASSP scores of the participants in the baseline-test, pre-test, post-test, and follow-up test.

did not changed for P1-Ben and P4-Roh, indicating that the nature of the designed protocol did not have a meaningful effect on this subscale.

\subsubsection{PSI-SF}

The PSI-SF questionnaire was filled in by the mothers to investigate the impact of our interventions on the mothers' stress (Figure 11). The result indicates that the stress of the mothers decreased considerably for three of the mothers from pre-test to post-test, while the scores remained in the same range in the follow-up test. The largest changes in the stress scores occurred for the mothers of P1-Ben and P4-Roh who had the least amount of hope/faith in their children's ability to learn. However, the effect size (Table 5) for this questionnaire is close to being "medium".

The parents were present in the therapeutic environment and observed their children's happiness/education process. Moreover, they sometimes were engaged in the games with their sons and played turn-taking group games. We believe that seeing the joy of the child could have a somewhat positive effect on the mother's stress. Being a playmate of the child (even for a couple of minutes) might foster the sense of parent-child relationship; therefore, the mother's feelings of usefulness improved as she helped her child do the tasks correctly. Moreover, the child's satisfaction obtained from attending the happy robotassisted program could increase his obedience to the parents in different areas and, as a result, increased the good feelings of the mother. In support of the PSISF's scores, Booth and Jernberg [42] mentioned that when the parents are an effective part of the treatment process, the children generalize the skills they learned to home and other environments. Contrary to the GARS and ASSP, the calculated effect size on the overall scores of the PSI-SF for both situations in Table 5 is approximately the same. Now, based on our observations and as a preliminary answer to the third research question, we indicated that robot-assisted

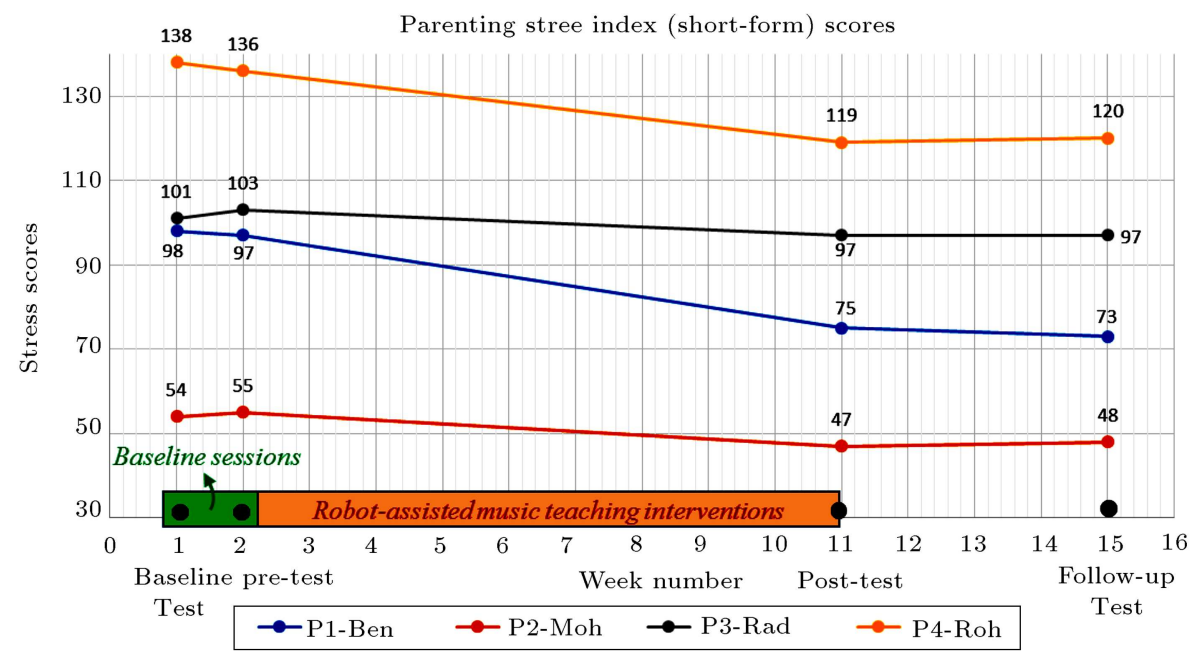

Figure 11. Total PSI-SF scores of the mothers in the baseline-test, pre-test, post-test, and follow-up test. 
music education interventions could decrease the stress level of the parents, howbeit with no large effect size.

\subsection{Interview with the parents}

The most important parts of the parents' claims are presented in the following paragraphs.

- P1-Ben: A decrease in hearing sensitivity was reported for P1-Ben by his mother. "Contrary to his past behavior, P1-Ben does not put his hands on his ears anymore when music/sound is playing". The mother claimed that she thought her son could not learn anything at all; however, her son was proven to have learned the notes and rhythms and she believed presence of the robot was the reason for this observation. "For the first time since attending pre-school, we observed that he had recently found a friend in his class". This educational intervention helped the mother feel a lot more relaxed. The mother felt that she was doing a great job for her son by bringing $\mathrm{P} 1-\mathrm{Ben}$ to this program (which mainly caused her stress level to be reduced).

- P2-Moh: The noteworthy factor for P2-Moh was the parents' high trust in our team and its educational-therapeutic interventions. P2-Moh's family was very satisfied for two reasons: first, the addition of running an educational program alongside the treatment and, second, teaching music which informed them of the potential abilities of their child in a new area (i.e., music). She added that "Nima plays an important role in his current paintings".

- P3-Rad: A slight improvement in P3-Rad's verbal skills was reported by his mother. "The music program and its positive effects were obviously different from his other treatment classes (e.g., ABA or speech therapy classes). P3-Rad is now trying to sing a song along with the background music; he showed less interest/tendency to do that before. I think increasing the time duration of the music classes would have had an even greater effect on a child like P3-Rad. The implicit teaching process through a cute robot increased my son's interest in participating in the class. P3-Rad's creativity increased somewhat and his descriptions and imaginary games have increased a bit".

- P4-Roh: The parents felt better about their son's treatment after all. "By bringing my son to this program, I am feeling more relaxed now. He has not seen such a cute robot anywhere else except in cartoons, and this obviously increased his excitement level. He is trying to speak more than before and we are excited about it. An overall comparison of his performance in the robot-assisted interventions with his ABA (Applied Behavioral Analysis) treatment sessions during the same time showed us that P4Roh could not have achieved the current progress without the robot".

\subsubsection{Subsection's summary}

Based on the mothers' overviews (acceptability of the study) in Subsections 3.4 and 3.5, it was observed that the autism severity and the parental stress of the subjects decreased slightly and the social skills of the children increased somewhat after the robotic-assisted music program. Of the most interesting outputs of this research, which is highly recommended to be studied deeply in the future, were the reported improvement in stereotyped behaviors of P4-Roh as well as verbal skills of P3Rad and P4-Roh. These two participants had more serious speech deficits than P1-Ben and P2-Moh did. This is in line with the idea that speech and music share the same neuronal network; therefore, teaching music should improve/affect verbal skills positively [28].

\subsection{Interview with the therapists}

At the beginning of the class and based on the WOZ robot control style, the therapists did not believe that the robot could play the assistant role very well; however, Nima's unpredictable and spontaneous dialogs/actions during the sessions forced the teachers to react appropriately and provided lots of creative situations for our music-teachers to help the children's learning process. In fact, Nima was totally accepted as an attractive/effective colleague by the therapists in the early sessions and the robot's presence could not be ignored. The therapists stated that "In traditional/classical music classes, the teacher uses his/her knowledge, experience, and influence to affect the students (i.e., parameters that Nima does not have now); however, what we observed in our work was that the effect of the robot's charm is very high. At the moment, the robot's performance in terms of reaction time as well as its communication are acceptable; however, it still cannot replace a human music teacher. While a similar progress rate was probably expected without using the robot, we are not sure if the participants would have been as happy/eager to attend the classes as they were with the robot. We also felt relaxed in Nima's company. All in all, social robots could be effective as facilitators in music classes for the children with ASD".

\subsubsection{Subsection's summary}

To answer the fourth research question, we indicated that robots not only could be quite acceptable in the role of facilitators in active music-teaching classes, but also seemed not have added any additional pressure on the teachers, too. Based on the unpredictable behaviors of children with $A S D$ and robots' autonomy issues, we 
believe that robots are not a suitable replacement for the human teachers, but they could be attractive coteachers.

\subsection{Results section's summary}

To answer the second research question, our robotassisted program could positively affect the social and cognitive skills of the participants in this study; however, in general, the effects of the music therapy sessions on participants' behaviors seem to be different for children from different points on the autism spectrum; even our high-functioning children showed different potential behavioral improvement. This may be due to the combinatorial nature of the developed games affecting various skills of the participants.

Among different arts, music can apply the high level of involvement/engagement between two brain's hemispheres [28]. Thanks to our designed scenario, we were able to provide a platform that enabled us to simultaneously influence diverse cognitive, social, and emotional behaviors of children with autism. Teaching music to children with autism is really a cognitivesocial task. However, the teaching efficiency does have several parameters, some of which are dependent on the personality, age, and talent of the subjects or even on the music background and families' cooperation. This case-study design research was done to obtain proof for the concept in a short-time and compact study and the potential of using social robots as facilitators in active music-based sessions. The positive points discussed in this preliminary exploratory study are promising and can shed light on continuing music therapy for children with autism using robotic technology.

\section{Application and implication of the study}

Using robots as a modern technology/tool in diverse educational/treatment classes will be unavoidable in the next few years and cognitive research; in addition, music communities will also experience this intervention approach. Cute characteristics of social robots would increase/facilitate effective engagement level and obedience of children with autism toward teachers/therapists and robots [21] in the music-based interventions. Assistant robots have the catalytic role in making the education [43,44] and social/cognitive rehabilitation process of children [21] in friendly environments appealing, and this could increase the attention span of the participants and their tendency to get deeply involved in the educational/treatment music tasks. Children receive the best education from those they like and not necessarily those with the best technical skills $[45,46]$. Robots could also benefit the music teachers, clinical supervisors, and music therapy educators in several ways such as being a great help for automatic/real-time treatment assessment as well as keeping record of the children's performance in the music sessions. We highly recommend practicing music-therapists and Human-Robot Interaction (HRI) researchers to continue a similar or even modified version of such studies widespread for children with mental disabilities in order to figure out comprehensive positive/negative aspects of using this technology in music education/therapy. The focus of our study was to show the utility/usefulness of the HumanRobotic Interaction as on e-step-ahead to improve traditional/classical music-based therapies (even across the different languages/cultures). Due to the findings presented in the literature $[23,24,37,47,48]$, our designed games could have the potential to positively affect a variety of cognitive skills of the children.

\section{Limitations of the study}

Although we tried our best to control the intervention situations, there is still a possibility that some of the improvements observed in music education and/or social skills are attributable to other interventions or education these participants may be receiving [29], which was obviously impossible to control this entirely. Moreover, we could not separate the effects of either factors, i.e., 1) the robot's attendance and 2) the music-based games, on the subjects' improvements, separately. In this research, we only considered the potential of the designed scenario as a case-study qualitative approach $[49,50]$, and no valid statistical analysis (e.g., paired T-test, ANOVA, etc.) could be scientifically done on the results. Moreover, the video coding results of the intervention sessions are not presented in this paper. All in all, we believe that the most important limitations in our study are: a) the small number of the participants as well as not having a control group, b) children's maturation, c) potential effects of the other classes outside our sessions, d) heterogeneous autism severity of the subjects, e) unpredicted behaviors of the subjects (specially the low-functioning participant) during the sessions, f) engineering technical issues, g) accessing valid tools to accurately measure children's behavior, and h) the small number of the sessions. At this point, we are still unable to answer the question of whether conducting the same scenarios with only a humantherapist (without robots) would be more/less effective than the robot-assisted interventions for participants with autism, and also figuring out the exact effect of such a rehabilitation method remains unanswered.

\section{Conclusion}

Through the designed music-based scenario package, the potential of music-based intervention on four children with ASD's improvement in learning music, social, 
and cognitive skills was explored. The strength of the proposed educational-therapeutic scenario was its hierarchical design. As an exploratory finding, it was indicated that assistant robots do have the ability to teach the fundamentals of music to children with highfunctioning autism. We also saw some improvement in fine hand imitation, using both hands in order, and rhythm identification for (especially two of) the highfunctioning participants. The high-functioning subjects can now all read/play the notes/simple musical sentences, and their progress was quite acceptable during the three months interventions. Nima's presence seemed to have appropriate effect on their willingness and increased the motivation of the children (especially the high-functioning subjects) to become deeply involved in the music education. The communication rate with Nima was observed to be quite remarkable, and the children took opportunities to induce the robot to speak with them. In the case of the low-functioning subject, some improvements in verbal skills and a decrease in stereotyped behaviors were observed. The parents of the autistic children felt better towards their sons, and the GARS showed that the autism severity of all of the participants was reduced somewhat after the intervention sessions. From the data reported by the psychologists as well as the ASSP questionnaire, some progress for the participants in social skills was observed. The children usually felt relaxed during the program, perhaps because the musical instruments used in this research were considered non-scary and non-stressful tools. The designed study procedures seemed to be feasible and acceptable in this primary stage.

\section{Acknowledgement}

Our profound gratitude goes to the "Center for the Treatment of Autistic Disorders (CTAD)" and its psychologists for their contributions to the clinical trials with the children with autism. This research was funded by the "Cognitive Sciences and Technology Council" (CSTC) of Iran (http://www.cogc.ir/). We also appreciate the Iranian National Science Foundation (INSF) for their complementary support of the Social \& Cognitive Robotics Laboratory (http://en.insf.org/).

\section{References}

1. Geretsegger, M., Holck, U., Bieleninik, Ł., and Gold, C. "Feasibility of a trial on improvisational music therapy for children with autism spectrum disorder", Journal of Music Therapy, 53(2), pp. 93-120 (2016).

2. Kim, J., Wigram, T., and Gold, C. "The effects of improvisational music therapy on joint attention behaviors in autistic children: A randomized controlled study", Journal of Autism and Developmental Disorders, 38(9), pp. 1758-1766 (2008).

3. Wan, C.Y., Bazen, L., Baars, R., et al. "Auditorymotor mapping training as an intervention to facilitate speech output in non-verbal children with autism: a proof of concept study", PloS one, 6(9), e25505 (2011).

4. Overy, K., Classroom Rhythm Games for Literacy Support, Music and Dyslexia: A Positive Approach, pp. 26-44 (2008).

5. Pouretemad, H.R., Diagnosis and Treatment of Joint Attention in Autistic Children (in Persian), Tehran, Iran: Arjmand Book (2011).

6. Bhat, A.N. and Srinivasan, S. "A review of 'music and movement' therapies for children with autism: embodied interventions for multisystem development", Frontiers in Integrative Neuroscience, 7(22), pp. 1-15 (2013).

7. Kern, P., Rivera, N.R., Chandler, A., and Humpal, M., "Music therapy services for individuals with autism spectrum disorder: A survey of clinical practices and training needs", Journal of Music Therapy, 50(4), pp. 274-303 (2013).

8. Kern, P., Whipple, J., Wakeford, L. et al., Early Childhood Music Therapy and Autism Spectrum Disorders: Developing Potential in Young Children and Their Families, Jessica Kingsley publishers (2012).

9. Lim, H.A. and Draper, E. "The effects of music therapy incorporated with applied behavior analysis verbal behavior approach for children with autism spectrum disorders", Journal of music therapy, 48(4), p. 532 (2011).

10. Corbett, B.A., Shickman, K., and Ferrer, E. "Brief report: the effects of Tomatis sound therapy on language in children with autism", Journal of Autism and Developmental Disorders, 38(3), pp. 562-566 (2008).

11. Kalas, A. "Joint attention responses of children with autism spectrum disorder to simple versus complex music", Journal of Music Therapy, 49(4), pp. 430-452 (2012).

12. Lanovaz, M.J., Fletcher, S.E., and Rapp, J.T. "Identifying stimuli that alter immediate and subsequent levels of vocal stereotypy a further analysis of functionally matched stimulation", Behavior Modification, 33(5), pp. 682-704 (2009).

13. Wood, S., "A study of the effects of music on attending behavior of children with autistic-like syndrome", Master's Thesis, San Jose State University (1991).

14. LaGasse, A.B. "Effects of a music therapy group intervention on enhancing social skills in children with autism", Journal of Music Therapy, 51(3), pp. 250-275 (2014).

15. Pasiali, V. "The use of prescriptive therapeutic songs in a home-based environment to promote social skills acquisition by children with autism: Three case studies", Music Therapy Perspectives, 22(1), pp. 11-20 (2004). 
16. Katagiri, J. "The effect of background music and song texts on the emotional understanding of children with autism", Journal of Music Therapy, 46(1), pp. 15-31 (2009).

17. Simpson, K., and Keen, D. "Music interventions for children with autism: narrative review of the literature", Journal of Autism and Developmental Disorders, 41(11), pp. 1507-1514 (2011).

18. Carnahan, C., Basham, J., and Musti-Rao, S. "A low-technology strategy for increasing engagement of students with autism and significant learning needs", Exceptionality, 17(2), pp. 76-87 (2009).

19. Taheri, A., Meghdari, A., Alemi, M., Pouretemad, H.R, Poorgoldooz, P., and Roohbakhsh, M. "Social robots and teaching music to autistic children: Myth or reality?", Lecture Notes in Computer Science (LNCS): Social Robotics, 9979, pp. 541-550 (2016).

20. Mavadati, S.M., Feng, H., Gutierrez, A., and Mahoor, M.H. "Comparing the gaze responses of children with autism and typically developed individuals in humanrobot interaction", In Humanoid Robots (Humanoids), 14th IEEE-RAS International Conference, pp. 11281133 (2014).

21. Scassellati, B., Admoni, H., and Mataric, M. "Robots for use in autism research", Annual Review of Biomedical Engineering, 14, pp. 275-294 (2012).

22. Soleiman, P., Salehi, S., Mahmoudi, M., Ghavami, M., Moradi, H. and Pouretemad, H.R. "RoboParrot: A robotic platform for human robot interaction, case of autistic children", Second RSI/ISM International Conference on Robotics and Mechatronics (ICRoM), IEEE, pp. 711-716 (2014).

23. Gifford, T., Srinivasan, S., et al. Using Robots to Teach Musical Rhythms to Typically Developing Children and Children with Autism, University of Connecticut (2011).

24. Peng, Y.H., Lin, C.W., Mayer, N.M., and Wang, M.L. "Using a humanoid robot for music therapy with autistic children", In Automatic Control Conference (CACS), International, IEEE, pp. 156-160 (2014).

25. Srinivasan, S.M., Eigsti, I.M., Neelly, L., and Bhat, A.N. "The effects of embodied rhythm and robotic interventions on the spontaneous and responsive social attention patterns of children with autism spectrum disorder (ASD): A pilot randomized controlled trial", Research in Autism Spectrum Disorders, 27, pp. 54-72 (2016).

26. https://www.softbankrobotics.com/emea/en (2018)

27. Taheri, A., Alemi, M., Meghdari, A., Pouretemad, H.R., Basiri, N.M., and Poorgoldooz, P. "Impact of humanoid social robots on treatment of a pair of Iranian autistic twins", Lecture Notes in Computer Science (LNCS): Social Robotics, 9388, pp. 623-632 (2015).

28. Zadeh Mohammadi, A., Music Education and Music Therapy for Exceptional Children (in Persian), Tehran, Iran, Kargah Nashr Publication (1995).
29. Kazdin, A.E., Single-Case Research Designs: Methods for Clinical and Applied Settings, Oxford University Press (2011).

30. Robb, S.L., "Reporting guidelines for musicbased interventions by Sheri L. Robb, Janet S. Carpenter and Debra S. Burns" (2010) doi: 10.1177/1359105310374781.

31. Gardner, H. "Children's duplication of rhythmic patterns", Journal of Research in Music Education, 19(3), pp. 355-360 (1971).

32. Mundy, P., Delgado, C., Block, J., Venezia, M., Hogan, A., and Seibert, J., Early Social Communication Scales (ESCS), Coral Gables, FL: University of Miami (2003).

33. Gilliam, J.E., Gilliam Autism Rating Scale: Examiner's Manual, Pro-ed, Austin, TX (1995).

34. Bellini, S. and Peters, J.K. "Social skills training for youth with autism spectrum disorders", Child and Adolescent Psychiatric Clinics of North America, 17(4), pp. 857-873 (2008).

35. Abidin, R.R., Parenting Stress Index-Short Form, Charlottesville, VA: Pediatric Psychology Press (1990).

36. Cohen, J., Statistical Power Analysis for the Behavioral Sciences, Revised Ed. (1977).

37. Peters, J.S., Music Therapy: An Introduction. Charles C Thomas Publisher (2000).

38. Finnigan, E. and Starr, E. "Increasing social responsiveness in a child with autism A comparison of music and non-music interventions", Autism, 14(4), pp. 321348 (2010).

39. Tapus, A., Peca, A., Aly, A., et al. "Children with autism social engagement in interaction with Nao, an imitative robot - A series of single case experiments", Interaction Studies, 13(3), pp. 315-347 (2012).

40. Feil-Seifer, D. and Matari, M.J. "Toward socially assistive robotics for augmenting interventions for children with autism spectrum disorders", In: Experimental Robotics: The Eleventh International Symposium. Springer, Berlin, pp. 201-210 (2009). DOI: 10.1007/978-3-642-00196-3_24.

41. Werry, I., Dautenhahn, K., Ogden, B., and Harwin, W. "Can social interaction skills be taught by a social agent? The role of a robotic mediator in autism therapy", In Cognitive Technology: Instruments of Mind, Springer Berlin Heidelberg, pp. 57-74 (2001).

42. Booth, P.B. and Jernberg, A.M., Theraplay: Helping Parents and Children Build Better Relationships Through Attachment-Based Play, John Wiley and Sons (2009).

43. Alemi, M., Meghdari, A., and Ghazisaedy, M. "The impact of social robotics on L2 learners' anxiety and attitude in English vocabulary acquisition", International Journal of Social Robotics, 7(4), pp. 523-535 (2015).

44. Taheri, A.R., Alemi, M., Meghdari, A., PourEtemad, H.R., and Basiri, N.M. "Social robots as assistants for autism therapy in Iran: Research in progress", Second 
RSI/ISM International Conference on Robotics and Mechatronics (ICRoM), pp. 760-766, IEEE (2014).

45. Alemi, M., Meghdari, A., Basiri, N.M., and Taheri, A. "The effect of applying humanoid robots as teacher assistants to help Iranian autistic pupils learn English as a foreign language", Lecture Notes in Computer Science (LNCS): Social Robotics, 9388, pp. 1-10 (2015).

46. Noor Mohammadi, F., "Effect of the orff music on improving rhythm structuring", (in Persian), Developmental Psychology Quarterly, Tehran (2003).

47. Taheri, A., Meghdari, A., Alemi, M., and Pouretemad, H.R. "Human-robot interaction in autism treatment: A case study on three pairs of autistic children as twins, siblings, and classmates", International Journal of Social Robotics, 10(1), pp. 93-113 (2018).

48. Taheri, A., Meghdari, A., Alemi, M., and Pouretemad, H.R. "Clinical interventions of social humanoid robots in the treatment of a set of high- and low-functioning autistic Iranian twins", Journal of Scientia Iranica, Transactions B: Mechanical Engineering, 25(3), pp. 1197-1214 (2018)

49. Brownell, M.D. "Musically adapted social stories to modify behaviors in students with autism: Four case studies", Journal of Music Therapy, 39(2), pp. 117-144 (2002).

50. Merriam, S.B. "Case study research in education: a qualitative approach", The Jossey-Bass Education Series, The Jossey-Bass Higher Education Series and The Jossey-Bass Social and Behavioral Science Series, San Francisco, CA, US: Jossey-Bass (1988).

\section{Biographies}

Alireza Taheri received his $\mathrm{PhD}$ in Mechanical Engineering with emphasis on Social Robotics at Sharif University of Technology, Tehran, Iran. He is a Post-Doctoral Research Associate at the Social and Cognitive Robotics Laboratory, Sharif University of Technology. During 2015-2017, he spent a total of oneyear sabbatical as a research scholar at Yale University and University of Denver, USA.

Ali Meghdari is a Professor of Mechanical Engineering at Sharif University of Technology (SUT) in Tehran. Professor Meghdari has performed extensive research in various areas of robotics: social \& cognitive robotics, mechatronics, bio-robotics, and modelling of biomechanical systems. He has been the recipient of various scholarships and awards, the latest being: The 2012 Allameh Tabatabaei Distinguished Professorship Award by the National Elites Foundation of Iran (BMN), the 2001 Mechanical Engineering Distinguished Professorship Award from the Ministry of Science, Research \& Technology (MSRT) in Iran, and the 1997 ISESCO Award in Technology from Morocco. He is currently the Director of the Centre of Excellence in Design, Robotics and Automation (CEDRA), an Affiliate Member of the Iranian Academy of Sciences (IAS), and a Fellow of the American Society of Mechanical Engineers (ASME).

Minoo Alemi received her $\mathrm{PhD}$ in Applied Linguistics (TEFL) from Allameh Tabataba'i University in 2011. She is currently an Assistant Professor and Division Head of Applied Linguistics in Islamic Azad University, West Tehran Branch. She is the Founder of Robot-Assisted Language Learning (RALL) and the co-founder of Social Robotics in Iran, which she achieved as a Post-Doctoral Research Associate at the Social \& Cognitive Robotics Laboratory of Sharif University of Technology. Her areas of interest include discourse analysis, interlanguage pragmatics, social robotics, and RALL. Dr. Alemi has been the recipient of various teaching and research awards from Sharif University of Technology, Allameh Tabataba'i University, Islamic Azad University, and Int. Conf. on Social Robotics (ICSR2014), Sydney-Australia. She has published over 75 papers and books in refereed national and international conferences, and journals. She is also on the editorial board of the British Journal of Educational Technology (BJET).

Hamid Reza Pouretemad is a Professor of Clinical Neuropsychology and the Founder and the Head of the Institute for Cognitive and Brain Sciences, Shahid Beheshti University, Tehran, Iran. He is also the founder of Iranian Society for Cognitive Science and Technology, and Director of the Center for the Treatment of Autistic Disorders (CTAD) in Tehran, Iran. He has done extensive research on children with autism spectrum disorder. He has published over 150 papers in refereed national and international conferences and journals. 